\title{
A Bright Future for Magnetic Resonance
}

\author{
Geoffrey Bodenhausen
}

This contribution seeks to identify a few factors that have contributed to the success of a thriving discipline, and to identify threats that could put future prospects at risk. Many of these factors are not specific to magnetic resonance, and may also apply to other areas of scientific research.

\section{Introduction}

Does magnetic resonance have a bright future? Ever since magnetic resonance in condensed phase started in 1945, questions about its future prospects (or its imminent doom) have been asked time and again. Some, like Nicolaas Bloembergen, left the field at an early stage because they felt there was no hope to gather another Nobel Prize after Bloch's and Purcell's. Perhaps the prizes given later to Ernst, Lee, Osheroff, Richardson, Wüthrich, Leggett, Mansfield and Lauterbur have had similar effects on some ambitious young people. But most players in the field have applauded these distinctions that strengthen the entire field. The fact is that - save perhaps a few specialties like mass spectroscopy for proteomics and X-ray diffraction for structural biology - there are few fields that can boast such a high visibility as magnetic resonance. This is, of course, largely due to the popularity of MRI as a diagnostic tool: everybody knows how powerful MRI can be, yet very few can explain how it works.

Since its birth 70 years ago, magnetic resonance has given rise to an astounding wealth of promising offshoots. It is hard to imagine that all of these offshoots would shrivel up and die out. Think of natural product chemistry. Think of drug screening. Think of structural biology. Think of the localization of mental activity by functional MRI. Think of the mapping of nerve bundles by diffusion tensor imaging (DTI). Think of metabolomics. Think of reaction mechanisms. Think of homogenous catalysis. Think of surface science. Think of quantum computing. Even if some of these applications may not bear fruit (or less than expected by ever-more-demanding granting agencies), other applications are likely to blossom. In truth, no prophet can tell how many novel offshoots will grow up in the next decades.

\section{Pitfalls}

There are some dangers looming around the corner. Magnetic resonance can only thrive in times of peace. Sabre-rattling does not help. Magnetic resonance can only flourish in a society that is not exclusively obsessed with making money. The current fascination for slippery financial 
deals and easy money does not help. Corruption, perhaps the best-distributed commodity in our increasingly lawless world, does not help. Magnetic resonance can occasionally stimulate the economy, but should not be viewed as a source of revenue. Expecting MBA's to give new orientations to industrial research does not help. Asking patent attorneys to acclaim the virtues of 'innovation' does not help. When governmental funding agencies attempt to channel grants to 'useful' research, this is often counterproductive. Magnetic resonance requires highly educated people. Lowering educational standards to cut government expenditure does not help. Unreasonable fear of elitist education does not help. Understandably, more and more young scientists are driven by a desire to boost their visibility and publish in flashy but superficial magazines. This does not help. Replacing class-room lectures by massively online open courses (MOOCs) does not help, except perhaps if there are no alternatives on hand.

\section{Instrument Companies and their MBA's}

A distinctive feature of magnetic resonance is the complementarity of academic and industrial research. Academic researchers often rely on industrial partners to develop new instruments they need. Conversely, many instrument companies have relied on academics to foster the development of novel paraphernalia. Many pioneers of NMR were deeply involved in joint developments: in the early days, Wes Anderson, Richard Ernst, Ray Freeman, and many others, enjoyed the support of scientifically literate management who did not recoil from risky innovation. Unfortunately, much of today's management appears amazingly ignorant about the 'core business' that they are supposed to manage.

Ray Freeman and Gareth Morris [1] recently wrote a vivid history of Agilent's fateful decision of 14th October 2014 to dismantle the NMR division they had bought from Varian Inc. for about $\$ 1.5$ billion in cash a few years earlier: "By closing down the whole NMR, MRI and magnet operation, Agilent has done a great disservice to a distinguished heritage and to a scientific community that had been faithful to Varian through thick and thin. [...] There remains an acute sense of loss, resentment, and even betrayal, not least at the lay-off of hundreds of former employees, many of whom were popular and respected members of the NMR family. [...] Ruthless American business administration methods that work for a shoe factory may be much less successful when applied to an enterprise like Varian NMR." [1].

Humour seems the best response. "A young MBA, suddenly placed in charge of a high technology group, understood perfectly well that there was only a year or two to 'turn things around' so naturally concentrated on short-term planning, with scant attention to the long-term future. It is $[\ldots]$ possible that a newcomer, trained purely in business doctrine, was not really comfortable dealing with unruly and insubordinate staff, armed with their superior knowledge 
of magnetic resonance. This kind of friction affected company harmony, and a few top scientists drifted away, or were even 'eased out' by the management. Like an inherently unstable heavy isotope, perhaps a nucleus made up of too many prima donnas is doomed to eventual disintegration?" [1].

At the time of writing, the question arises if remaining companies like Bruker, JEOL, Siemens, Philips and General Electric (GE) will do better than Agilent. Some believe that only small startup companies can take ground-breaking initiatives. However, truly creative scientists are not necessarily keen on start-ups: they have little patience with financial wizards who expect 'business plans' and other 'visionary' documents. A new generation of ambitious go-betweens now thrives at the interface between creative scientists and poorly educated bankers. It is to be feared that these go-betweens will boost the growing ranks of unproductive parasites of today's economy.

Rumour has it that corporations such as GE employ more lawyers that engineers. Not surprisingly, their foresight leaves much to be desired: developing and marketing a machine that can inject sterile solutions of hyperpolarized pyruvate into patients who suffer from prostate cancer is undeniably a great achievement [2], but such a bold enterprise will only become truly successful if it can build on a broad effort in basic research on hyperpolarized samples and preclinical MRI studies on animals.

\section{Shortcomings of Science Policy}

We have written a few vitriolic papers [3-5] against the widespread use of ill-conceived impact factors, and more generally against the seemingly blind confidence in 'bibliometrics', i.e., the mindless bean-counting of numbers of citations. In truth, it hardly matters if some scientists derive a narcissistic satisfaction from the impact of their papers, nor does it matter if they enjoy watching their bulging muscles in the mirrors of noisy gyms. However, it does matter if granting agencies, universities, faculties and institutes use impact factors of journals, numbers of citations, $\mathrm{H}$-factors and the like to guide decisions about appointments, scholarships and funding. There seems to be a devilish interplay of at least five toxic factors that form a vicious circle: (i) the greed of publishers, (ii) the obsession of some editorial boards to boost their impact factors, (iii) the laziness of granting agencies and university management, (iv) the narcissism of senior authors, and, last but not least, (v) the legitimate concern of graduate students and post-doctoral fellows to be rewarded for their efforts. Understandably, many young scientists believe that their chances to secure attractive jobs will be improved by being first author on some flashy paper in a popular bling-bling magazine. It is obviously hard to ignore such expectations. I myself must plead guilty to having contributed to this vicious circle. 
In my view, our only hope to break out of this vicious circle is that governments such as those of India, Brazil, China, possibly the European Union, and perhaps USA challenge the oligopoly of publishers. The latter make fantastic profits by taking advantage of the public sector, through outrageous fees charged to libraries, by demanding that all intellectual property generated by publicly-funded laboratories be transferred to them, and by exploiting scientists as authors, as reviewers, and finally as proof-readers. Not to mention those who work for some ludicrous fees as members of editorial boards. Publishers have a maddening habit of introducing errors into manuscripts that the authors must painstakingly remove. Private publishers have bought up a great deal of intellectual property of respectable journals. They scornfully refer to classic papers as 'back copies', and sell them for exorbitant fees. Today, the main service that publishers provide appears to be the insertion of volume and page numbers on manuscripts! Surely, this parasitical modus vivendi cannot be sustainable in the long run. Once the above-mentioned vicious circle that threatens our intellectual endeavour is discontinued, basic research in general, and fundamental magnetic resonance in particular, will be able to blossom again without obstruction.

\section{The Solace of Creativity}

The transformative power of some inventions is truly staggering. But not everyone can expect to invent a steam engine or a transistor. Not all crazy ideas are worth following up. The important thing is to keep trying. One of the specific aspects of magnetic resonance is that novel ideas can be tried out very quickly. With some exceptions like dynamic nuclear polarization $(\mathrm{DNP})^{1}$, there is usually little need to build elaborate equipment. This gives the field of magnetic resonance a mercurial and, at times, volatile character.

How does innovation work in practice? We can draw a few telling illustrations from magnetic resonance. Consider modern MRI as it is now practiced in numerous hospitals around the world, and not least in India. The work by Anil Kumar, Dieter Welti and Richard Ernst [6] apparently resulted from the convergence of two seemingly innocuous fields of research. Paul Lauterbur was tinkering with NMR spectra observed in the presence of a magnetic field gradient [7], and realized that an image could be reconstructed by back-projection, itself a fashionable idea at that time because of the development of X-ray scanning tomography. At about the same time, Richard Ernst was exploring possible applications of the concept of two-dimensional Fourier transformation (2D-FT), in part inspired by a near-mythical lecture that Jean Jeener had delivered a few years before at an obscure summer school [8]. When I was an undergraduate student at ETH in 1974, I had the fortune to attend a group seminar given by Richard Ernst after

${ }^{1}$ DNP allows one to enhance NMR signals by saturating the transitions of electron spins by microwave irradiation. 
returning from a meeting. I was quite naïve at the time, but I'm quite sure that nobody in the room (except perhaps the speaker) perceived that we were witnessing the birth of Fourier MRI. A truly great idea indeed!

Closer to our time, I recall how Philippe Pelupessy at the École Normale Supérieure contributed what I believe to be a revolutionary idea to the field of MRI, called 'cross-encoding' [9]. Pelupessy was intrigued by the innovative work of Lucio Frydman [10], who had been inspired by an idea known as echo-planar imaging $(E P I)^{2}$ that had been invented by Peter Mansfield and co-workers [11] to obtain 2D images in a fraction of a second. Lucio Frydman carried this idea from 2D MRI to in vitro 2D spectroscopy, to correlate chemical shifts of, say, protons and nitrogen-15 nuclei, instead of spatial co-ordinates as in EPI. Frydman's method has come to be known as 'single-scan 2D' or 'ultrafast 2D' [10]. Pelupessy quickly realized that adiabatic pulses [12] would be much more efficient for encoding the phases of the magnetization than the frequency-shifted pulses that Frydman had proposed. Not satisfied with this contribution, Philippe Pelupessy and Raphael Paquin understood that the idea of 'single-scan 2D' could be carried back from in vitro 2D spectroscopy to imaging. The resulting 'cross-encoding' [9] method has the remarkable (and perhaps, in part, serendipitous) advantage that one can record high-resolution images of an object immersed in an inhomogeneous magnetic field, or, more importantly, of an object that suffers from internal field gradients because of discontinuities in its magnetic susceptibility. In my admittedly biased view, this is perhaps the most fundamental step since the Fourier MRI paper by Anil Kumar et al [6]. I was strengthened in my optimism by a rare compliment of the late Paul Callaghan. Unfortunately, the MRI community does not seem to have paid much attention to this idea. So far, in the 5 years since it has been published, the 'cross-encoding' paper has only been cited twice (disregarding a single self-citation), a disheartening number even for those who like to challenge the triumphant march of beancounters. Incidentally, the paper appeared in the Journal of Magnetic Resonance, after being rejected by several pretentious (and ill-managed) magazines such as Science and Nature. Perhaps the modest impact factor of the Journal of Magnetic Resonance is to blame for the lack of recognition of Pelupessy's invention.

A third illustration of how ideas bounce back and forth comes from our work on long-lived states (LLS) ${ }^{3}$ that were invented by Levitt and co-workers [13]. Populations that are locked in anti-symmetric singlet states can have surprisingly long lifetimes. New experiments were designed and new molecules were synthesized in the hope of extending these lifetimes ever more. However, it became suddenly apparent that the reduction of these lifetimes upon binding

\footnotetext{
${ }^{2} \mathrm{EPI}$ allows one to record two-dimensional images in a fraction of a second.

${ }^{3}$ LLS are spin states with populations that have long life-times.
} 
of suitable LLS-carrying drug molecules to proteins offered a novel tool to determine the stability of drug/protein complexes [14]. To our surprise, we suddenly found ourselves working hand-in-hand with pharmaceutical industry!

Maurice Goldman wrote about the so-called Pake doublets ${ }^{4}$ that arise from protons of water molecules trapped in solid gypsum. This discovery, made as far back as 1948, had a considerable impact because it gave direct evidence of the quantum-mechanical nature of nuclear moments: two proton spins can be found in parallel or antiparallel configurations, with populations that have vastly different lifetimes [15]. This spurred our current interest in the preparation and preservation of para-water, which we believe to have a long lifetime because of the antiparallel orientation of its two protons.

We could tell many more stories about innovation and creativity. Louis Pasteur, the first professor of chemistry at the École Normale Supérieure in Paris, wrote somewhere: Le hasard ne favorise que les esprits préparés: chance favours only prepared minds.

\section{The Strength of Our Community}

There are now many well-staffed and well-equipped facilities that are dedicated to magnetic resonance, not only in Europe and the USA, but also in Japan, China, and of course, India. The vitality of the community can be assessed though the frequency and popularity of meetings such as ISMAR, ICMRBS, ISMRM, EUROMAR, ENC, not to mention a myriad of specialized meetings on imaging, on solid-state NMR (Rocky Mountains, Chamonix), on porous materials, on hyperpolarized gases, on dynamic nuclear polarization, etc. In addition, there are a host of national meetings such as the Conference of the National Magnetic Resonance Society, India that gathered in Amritsar in March 2015.

The popularity of meetings is not the only hallmark of our vibrant community. It is a rewarding experience to see how new generations emerge, to watch how young people become familiar with subtle concepts of magnetic resonance, and how they gradually take over leadership from older generations. There can hardly be a greater pleasure than watching young students beaming with self-confidence when delivering lectures at challenging meetings.

\section{Keep Up the Standards!}

One of the great strengths of NMR and MRI is that we know what we measure, and that we know it very well. The fundamentals of the theory are very solid; almost everything can be found

\footnotetext{
4 'Pake doublets' are observed in magnetic resonance of solids with dipole-dipole couplings.
} 
in 'classic' papers by Bloch, Purcell, Redfield, Pake, Andrew, Overhauser, Slichter, McConnell, Gutowsky, Abragam, Goldman, Waugh, Mansfield, Lauterbur, Ernst, and many others. For a newcomer to the field of NMR, it may require years to digest such treasures of wisdom. Much of what we do today builds on the vision of the pioneers, by making good use of the vastlyimproved computational power, and by performing sophisticated experiments that were impossible in the old days. How many scientific communities share the privilege of such solid conceptual foundations?

But we should remain watchful. Is the current generation of $\mathrm{PhD}$ advisors doing a good job in transmitting the conceptual foundations? One sometimes comes to realize that graduate students (particularly in laboratories that focus on medical and biochemical applications) may not pay sufficient attention to fundamental aspects. Could this be another consequence of the shortcomings of science policy?

Jean Jeener recently commented how Thomas Baumann, a PhD student in Richard Ernst's group in 1971, carefully made notes while listening to Jeener's now-famous lecture at a summer school in Basko Polje [8]. Baumann's notes represent a testimony of what was actually said and shown at this meeting. If these notes still exist and are remarkably complete, this is not only a tribute to Baumann's habit of taking careful minutes of conferences, but it also reflects the old tradition of properly 'teaching' sophisticated concepts at schools by spending half an hour or so on a single idea, illustrated by no more than two or three slides. Today's galloping rhythm of flashy lectures, with many overcrowded slides per minute, essentially prevents the audience from taking any notes at all.

A meeting that has played an essential role for the emergence of new concepts was the Gordon Conference on magnetic resonance, which used to be held in New Hampshire every other year. It was discontinued when the number of participants dropped below 100. Could this be a sign that the emphasis is shifting to more specific applications, at the expense of fundamental concepts?

As Maurice Goldman wrote, freely translated from French: "NMR has expanded beyond the realm of mere specialists, which has important consequences. On the one hand, the methods that make the discipline so exciting require a highly sophisticated theoretical framework to be conceived and understood. On the other hand, the users of the resulting NMR methods [...] do usually not possess the background in physics that is required to properly understand what they are doing. This necessarily leads to a specialization between NMR experts who invent methods, and users who follow recipes. However, the dichotomy is never complete. The users must have some idea what happens when they apply the recipes, and the method designers benefit from the 
curiosity of the users who motivate and encourage them, otherwise their endeavour might boil down to a sterile game. This situation is far from unique in science, and can also be found in lowtemperature physics, for example. NMR is and will remain a highly elaborate and complex discipline, where two types of scientists, designers and users, must by necessity live together and collaborate to enhance the value of each others' efforts". [...] "NMR, now mostly a tool [...] for chemistry and medicine, is essentially a discipline of physics. Each of its developments necessitated a complex and mostly abstract theoretical elaboration, which however lead, sideby-side with fundamental advances in physics, to an enormous variety of practical uses, whose underlying basis is not always mastered by their practitioners. However, it works!" [15].

Let us quote Freeman and Morris once more: "It is surely impossible to conceive of chemistry today without NMR, and the later development of magnetic resonance imaging owes a great deal to its predecessor" [1].

\section{Conclusions}

This paper will hardly be cited, surely not boost the author's H-factor [16], and barely enhance his chances of success when submitting future research proposals. So one may wonder what drove him to write. Fortunately, there are other factors at work: the hope of motivating new generations of students in India and elsewhere, of warning them against pitfalls, and simply telling them how research can be a source of joy and satisfaction. Beyond the hope of motivating new generations, I would like to express my friendship for my Indian colleagues, my admiration for their creativity, and my respect for their vast culture.

\section{Acknowledgements}

The author is indebted to Jean Jeener, Maurice Goldman, Nicola Salvi, and Antoinette Molinié for constructive suggestions, and to the ENS and the EPFL for shelter and support.

\section{Suggested Reading}

[1] R Freeman and G A Morris, The varian story, J. Magn. Reson., Vol.250, pp.80-84, 2015.

[2] S J Nelson, J Kurhanewicz, D B Vigneron, P E Z Larson, A L Harzstark, M Ferrone, M van Criekinge, J W Chang, R Bok, I Park, G Reed, L Carvajal, E J Small, P Munster, V K Weinberg, J -H Ardenkjaer-Larsen, A P Chen, R E Hurd, L -I Odegardstuen, F J Robb, J Tropp, J A Murray, Metabolic imaging of patients with prostate cancer using hyperpolarized [1-C-13]pyruvate, Science Translat. Med., Vol.5, 198ra108, 2013.

[3] A Molinié and G Bodenhausen, Bibliometrics as weapons of mass citation; La bibliométrie comme arme de citation massive, Chimia, Vol.64, pp.78-89, 2009.

[4] Idem, English translation, Bunsen-Magazin, Vol.5, pp.188-198, 2010.

[5] A Molinié and G Bodenhausen, On toxic effects of scientific journals, J. Biosc., Vol.38, pp.189-199, 2013.

[6] Anil Kumar, D Welti, R R Ernst, NMR Fourier zeugmatography, J. Magn. Reson., Vol.18, pp.69-83, 1975. 
[7] P C Lauterbur, Image formation by induced local interactions: examples employing nuclear magnetic resonance, Nature, Vol.242, pp.190-191, 1973.

[8] J Jeener, Ampere International Summer School, Basko Polje, Yugoslavia, 1971.

[9] R Paquin, P Pelupessy, and G Bodenhausen, Cross-encoded magnetic resonance imaging in inhomogeneous fields, J Magn. Reson., Vol.201, pp.199-204, 2009.

[10] L Frydman, T Scherf and A Lupulescu, The acquisition of multidimensional NMR spectra within a single scan, Proc. Natl. Acad. Sci., USA, Vol.99, pp.15858-15862, 2002.

[11] M Stehling, $R$ Turner and $P$ Mansfield, Echo-planar imaging: magnetic resonance imaging in a fraction of a second, Science, Vol.254, pp.43-50, 1991.

[12] P Pelupessy, Adiabatic single scan two-dimensional NMR spectroscopy, J. Am. Chem. Soc., Vol.125, pp.12345$12350,2003$.

[13] M Carravetta, O G Johannessen and M H Levitt, Beyond the T1 limit: singlet nuclear spin states in low magnetic fields, Phys. Rev Lett., Vol.92, 153003-1-4, 2004.

[14] N Salvi, R Buratto, A Bornet, S Ulzega and I Rentero Rebollo, A Angelini, C Heinis and G Bodenhausen, Boosting sensitivity of ligand-protein screening by NMR of long-lived states, J. Am. Chem. Soc., Vol.134, pp.11076-11079, 2012.

[15] M Goldman, Histoire de la RMN, L'actualité chimique, Vol.273, No.204, pp.57-60 (translated by GB), 2004.

[16] R R Ernst, The follies of citation indices and academic ranking lists - A brief commentary to 'Bibliometrics as weapons of mass citation', Chimia, Vol.64, p.90, 2009; Bunsen-Magazin, Vol.5, p.199, 2010.

Geoffrey Bodenhausen ${ }^{a, b, c, d}$

${ }^{a}$ École Normale Supérieure-PSL Research University, Département de Chimie, 24 Rue Lhomond, 75005 Paris, France ${ }^{b}$ Sorbonne Universités UPMC Paris 6, 4 place Jussieu, 75005 Paris, France ${ }^{c}$ CNRS, UMR 7203 LBM, 75005 Paris, France

${ }^{d}$ Institut des Sciences et Ingénierie Chimiques, Ecole Polytechnique Fédérale de Lausanne, 1015 Lausanne, Switzerland Email: geoffrey.bodenhausen@ens.fr

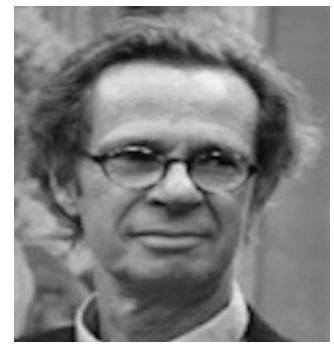

Geoffrey Bodenhausen, Diploma, ( ETH, Zürich, with R R Ernst), PhD (Oxford,UK with Ray Freeman); is Professor of Chemistry in Ecole Normale Supérieure, Paris, and in Ecole Polytechnique Fédérale de Lausanne in Switzerland. He specializes in methodological developments in NMR, has published 350 papers and has 24 patents. He is a co-author of the famous book on One \& Two-Dimensional NMR by Ernst and Wokaun. 